https://doi.org/10.11646/phytotaxa.358.3.8

\title{
Crespo, M.B., Martínez-Azorín, M. \& Mavrodiev, E.V. (2015) Can a rainbow consist of a single colour? A new comprehensive generic arrangement of the 'Iris sensu latissimo' clade (Iridaceae), congruent with morphology and molecular data.
} Phytotaxa 232: 1-78.

The passage from the page 4:

"This matter is however fairly problematic (Mavrodiev 2010, Mavrodiev et al. 2014), since Iris s.1. is to be widened to include plants with actinomorphic open flowers such as those in Belamcanda, not only plants with the typically 3-meranthic and frequently closed Iris-flower structure (Weberling 1989: 90) which constantly show "standards" (outer tepals), "falls" (inner ones) and petaloid-laminar style branches (i.e, Lawrence 1953, Mathew 1989a, Weberling 1989)"

\section{Should read:}

"This matter is however fairly problematic (Mavrodiev 2010, Mavrodiev et al. 2014), since Iris s.l. is to be widened to include plants with actinomorphic open flowers such as those in Belamcanda, not only plants with the typically 3meranthic and frequently closed Iris-flower structure (Weberling 1989: 90) which constantly show "standards" (inner tepals), "falls" (outer ones) and petaloid-laminar style branches (i.e, Lawrence 1953, Mathew 1989a, Weberling 1989, Mavrodiev \& Alexeev 2003: 51)" 